\title{
Development of a software to simulate effluent characteristics of textile wastewater treatment
}

\begin{abstract}
The composition of textile wastewaters is extremely varied due to the large spectrum of dyes and chemicals used in wet process resulting in greater variability in the success of different treatment processes. A software was developed to determine the required advanced oxidation process after biological treatment and to evaluate the possibility of applying advanced oxidation processes to any textile treatment plant. Regression equations related to the formulation of the process treatment options were successfully developed into a software program called TexTreat with integrated graphic user interface (GUI) component. TexTreat has the ability to determine the best option of advanced oxidation processes (AOPs) and predict characteristics of wastewater discharge in different retention times with an overall simulation accuracy of more than $89 \%$. Validation of the process treatment options and TexTreat display their applicability to utilise with different textile wastewater plants.
\end{abstract}

Keyword: Textile wastewater; Advanced oxidation process; AOP; Wastewater treatment; Effluent simulation; Biological treatment; Process treatment options 